\begin{tabular}{|c|c|}
\hline Title & Identification of Magnetization Characteristics of Material From Measured Inductance Data \\
\hline Author(s) & Maruo, A kito; Igarashi, Hajime; Sato, Y uki; Kawano, Kenji \\
\hline Citation & $\begin{array}{l}\text { IEEE Transactions on Magnetics, 55(6), } 7300205 \\
\text { https://doi.org/10.1109// MA G.2019.2896187 }\end{array}$ \\
\hline Issue Date & 2019-06 \\
\hline Doc URL & http:/hdl.handle.net/2115/74696 \\
\hline Rights & $\begin{array}{l}\text { C } 2019 \text { IEEE. Personal use of this material is permitted. Permission from IEEE must be obtained for all other uses, in } \\
\text { any current or future media, including reprinting/republishing this material for advertising or promotional purposes, } \\
\text { creating new collective works, for resal e or redistribution to servers or lists, or reuse of any copy righted component of } \\
\text { this work in other works. }\end{array}$ \\
\hline Type & article (author version) \\
\hline File Information & CEFC_0163.pdf \\
\hline
\end{tabular}

Instructions for use 


\title{
Identification of Magnetization Characteristics of Material from Measured Inductance Data
}

\author{
Akito Maruo $^{1}$, Hajime Igarashi ${ }^{1}$, Yuki Sato ${ }^{2}$, Kenji Kawano ${ }^{2}$, IEEE Member \\ ${ }^{1}$ Graduate School of Information Science and Technology, Hokkaido University, Sapporo 060-0814, Japan \\ 2 Texas Instruments Japan Limited, Tokyo 160-8366, Japan
}

\begin{abstract}
This paper introduces a novel identification method of the magnetization properties of the core material for an inductor from measured inductance data. The proposed method allows us to obtain the magnetic hysteresis characteristics without a special measurement instrument for material BH properties. The proposed method determines the parameters included in the distribution function for the Preisach model from the measured $L-I$ characteristics. It is shown that the identified initial magnetization curve and the minor loops are in good agreement with the original BH curves. The uniqueness for the identification problem is numerically verified.
\end{abstract}

Index Terms - Genetic Algorithm, Inductor, Inverse Problem, Magnetic Hysteresis, Preisach Model.

\section{INTRODUCTION}

$\mathrm{F}_{\mathrm{i}, ~}$ NITE element (FE) analysis of power inductors and reactors is based on the initial BH curves or hysteresis characteristics [e.g., 1]. It is, however, not always possible to obtain such data because a special measurement instrument for $\mathrm{BH}$ characteristics of magnetic material is necessary [2-4]. Moreover, it is sometimes hard to generate sufficiently strong magnetic field to measure the magnetic saturation properties. On the other hand, it is not difficult to measure the macroscopic properties of power inductors; the dependence of inductance on the DC-bias current and amplitude of the imposed AC current can be measured using, e.g., a network analyzer. It is remarkable that magnetic saturation can occur near the coil edges in an inductor core even when a weak current is imposed. Moreover, the measured inductance is attributed to the distributed magnetic field that changes in time to draw major or minor loops in the inductor core. From these observations, a question arises; is it possible to identify the magnetization characteristics of the core material used in an inductor from the measured inductance data?

In this paper, we propose a method to identify the $\mathrm{BH}$ characteristics including magnetic hysteresis from the measured dependence of the inductance of an inductor on the amplitude and bias of the imposed current. The hysteresis property is assumed to be described by the Preisach model with a distribution function while the proposed method can be extended to other hysteresis models such as Play model. The parameters included in the distribution function are determined by the genetic algorithm (GA) so that the measured inductance properties are reproduced. We discuss the accuracy of the identified $\mathrm{BH}$ characteristics and uniqueness for the identification.

Manuscript received April 1, 2015; revised May 15, 2015 and June 1, 2015; accepted July 1, 2015. Date of publication July 10, 2015; date of current version July 31,2015 . (Dates will be inserted by IEEE; "published" is the date the accepted preprint is posted on IEEE Xplore ${ }^{\circledR}$; "current version" is the date the typeset version is posted on Xplore $\left.{ }^{\mathbb{P}}\right)$. Corresponding author: A. Maruo (e-mail: maruo@em.ist.hokudai.ac.jp).

Digital Object Identifier (inserted by IEEE).

\section{NUMERICAL METHOD}

\section{A. $\quad$ Preisach model}

The Preisach model [5-7] is one of the hysteresis models which are widely used in FE analysis. In the Preisach model, the magnetic characteristic is represented by the superimposition of the basic hysteresis loops as shown in Fig. 1, where $H_{u}$ and $H_{v}$ are the upper and lower thresholds of the basic hysteresis loop. The magnetization $M$ becomes positive when $H$ increases to become greater than $H_{u}$. Similarly, $M$ becomes negative when $H$ decreases to become smaller than $H_{v}$. The Preisach model expresses $M$ in the following form:

$$
M=\iint_{D} K\left(H_{u}, H_{v}\right) d H_{u} d H_{v}+M_{\min }
$$

where $K$ and $M_{\min }$ denote the distribution function and the minimum value of $M$, respectively. Moreover, $D$ is a triangular domain in the Preisach plane shown in Fig.2 which satisfies $-H_{s} \leq H_{v} \leq H_{u} \leq H_{s}$. When the interval $\left[-H_{s}, H_{s}\right]$ is divided into $N_{p}$ subintervals $\left[H_{n}, H_{n-1}\right]\left(0<n<N_{p}-1\right)$, the integral in (1) is partitioned into

$$
K^{\prime}(i, j)=\int_{H_{j}}^{H_{j+1}} \int_{H_{i}}^{H_{i+1}} K\left(H_{u}, H_{v}\right) d H_{u} d H_{v}
$$

Then, (1) is rewritten for numerical evaluation as

$$
M=\sum_{i=0}^{N_{p}-1} \sum_{j=0}^{i} K^{\prime}(i, j) D(i, j)+M_{\text {min }}
$$

where $D(i, j)$ is defined by

$D(i, j)=\left\{\begin{array}{l}1: \text { positive magnetization } \\ 0: \text { negative magnetization }\end{array}\right.$

The initial magnetization curve as well as the major and minor $\mathrm{BH}$ loops can be expressed using the Preisach model. The AC magnetic property, shown in Fig. 3, of the material used for an inductor is computed from the slope of the minor loops governed by the Preisach model for the biased magnetic field $H$ as shown in Fig. 4. 


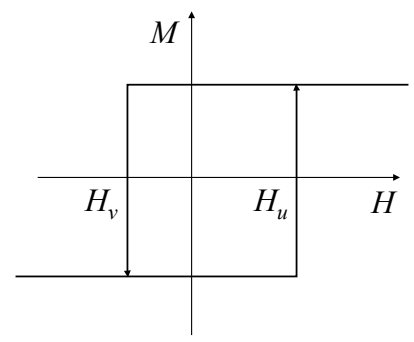

Fig. 1. Basic hysteresis loop

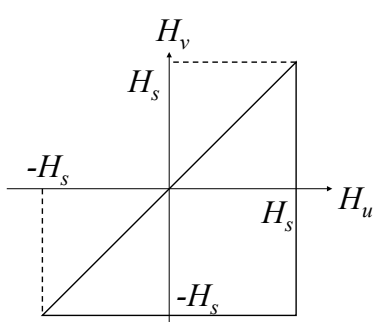

Fig. 2. Preisach plane

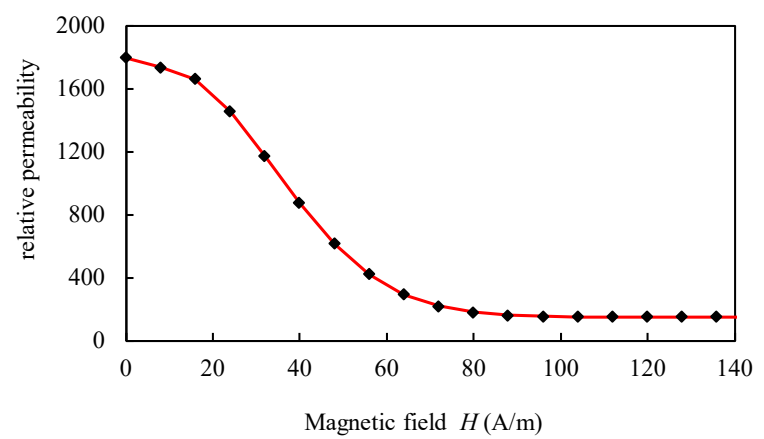

Fig. 3. AC magnetic property for biased magnetic field

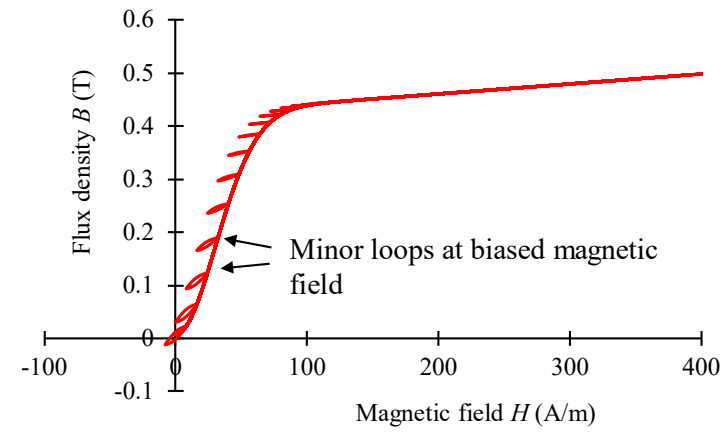

Fig. 4. Initial magnetization curve and minor loops

\section{B. Analysis of inductance}

We evaluate the inductance of an inductor by FE analysis in which the following magnetostatic equation is discretized:

$$
\operatorname{rot}(v \operatorname{rot} \boldsymbol{A})=\boldsymbol{J}
$$

where $v, \boldsymbol{A}$ and $\boldsymbol{J}$ denote the reciprocal of magnetic permeability, vector potential and current density, respectively. After the solution of the FE equation, the inductance $L$ is computed. When considering magnetic saturation, we may define inductance from the derivative $d \Phi / d I$ or the average $\int \Phi(I) I / I d I$. The measured inductance depends on the definition of inductance which differs for each measurement instrument. Here, for simplicity, we compute the AC inductance $L_{a c}$ using the peak value $I_{\text {peak }}$ of an AC current from

$$
L=\frac{\int_{\Omega} \boldsymbol{A} \cdot \boldsymbol{J} d \Omega}{I^{2}}
$$

On the other hand, the DC inductance $L_{d c}$ is computed by the following procedure:

(i) The magnetic flux density $\boldsymbol{B}$ is computed at each finite element for a bias current $I_{\text {bias }}$ using the nonlinear FE analysis based on the initial magnetization curve. (ii) For the magnitude of the magnetic flux density $\boldsymbol{B}$ at each finite element, the magnetic permeability is computed from Fig. 3.

(iii) Assuming a small input current, the linear FE analysis is performed using the magnetic permeability evaluated in step (ii), and $L_{d c}$ is computed from (6).

\section{IDENTIFICATION METHOD}

In this study, the BH characteristic of the material used by an inductor is identified from the inductance values, $L_{a c}$ and $L_{d c}$ defined in II, using GA, the detail of which is described in [7]. The distribution function $K\left(H_{u}, H_{v}\right)$ in (1) is here expressed by the Gaussian function with an elliptic exponent that includes three parameters $A, \sigma_{1}$ and $\sigma_{2}$ as follows:

$$
K=\frac{A}{2 \pi \sigma_{1} \sigma_{2}} \exp \left[-\frac{\left(\frac{H_{u}-H_{v}}{2}\right)^{2}}{2 \sigma_{1}^{2}}-\frac{\left(\frac{H_{u}+H_{v}}{2}\right)^{2}}{2 \sigma_{2}^{2}}\right]
$$

The magnetic flux density $\boldsymbol{B}$ is expressed by

$$
\boldsymbol{B}=\mu_{0} \mu_{r}^{\text {out }} \boldsymbol{H}+\boldsymbol{M}
$$

where $\mu_{r}^{\text {out }}$ is the relative permeability in the fully saturated region and $\boldsymbol{M}$ is obtained by the Preisach model based on (7). We determine the unknown parameters $\left(A, \sigma_{1}, \sigma_{2}, \mu_{r}^{\text {out }}\right)$ in (7) and (8) by solving the optimization problem defined by

$$
\begin{aligned}
\min F=\frac{1}{N_{i}} \sum_{i}^{N_{i}}( & \left.\frac{L_{d c}(i)-L_{d c}^{0}(i)}{L_{d c}^{0}(i)}\right)^{2} \\
& +\frac{1}{N_{j}} \sum_{j}^{N_{j}}\left(\frac{L_{a c}(j)-L_{a c}^{0}(j)}{L_{a c}^{0}(j)}\right)^{2}
\end{aligned}
$$

where $i, j$ run over the number of sampling points $N_{i}, N_{j}$, and $L_{d c}^{0}$ and $L_{d c}$ represent the original and computed DC inductances, respectively, and the same convention is used for the $\mathrm{AC}$ inductance. The cost function defined in (9) is minimized by GA for a set of optimization variables $A, \sigma_{1}, \sigma_{2}, \mu_{r}^{\text {out }}$, in which the fitness is evaluated as follows:

(i) The initial magnetization curve and $\mathrm{AC}$ magnetic property shown in Fig. 3 for the current parameters $\left(A, \sigma_{1}, \sigma_{2}, \mu_{r}^{\text {out }}\right)$ are computed using the Preisach model based on (7) and (8).

(ii) The $L_{a c}-I_{\text {peak }}$ and $L_{d c}-I_{\text {bias }}$ characteristics are computed using the method mentioned in II.B.

(iii) The cost function $F$ that measures the difference between the computed and original inductance values, $L_{d c}-L_{d c}^{0}$ and $L_{a c}-L_{a c}^{0}$, is evaluated by (9).

In GA, the individuals have a different set of $A, \sigma_{1}, \sigma_{2}, \mu_{r}^{\text {out }}$ that correspond to the $L_{a c}-I_{\text {peak }}$ and $L_{d c}-I_{\text {bias }}$ characteristics. They evolve to reproduce the original inductance characteristics. 


\section{NUMERICAL RESULT}

\section{A. Preparation of Inductance Characteristics}

We consider here the ferrite inductor shown in Fig. 5 for a numerical example. Table I summarizes the specific parameters of the inductor. The measured major $\mathrm{BH}$ curve of ferrite used for the magnetic core is shown in Fig. 6. The initial magnetization curve obtained from Fig. 6 is used to compute $L_{a c}-I_{\text {peak }}$ characteristic. Moreover, we determine the parameters $\left(A, \sigma_{1}, \sigma_{2}, \mu_{r}^{\text {out }}\right)$ in (7) and (8) from the measured curve in Fig. 6 by the curve fitting that solves

$$
\min E=\frac{1}{N_{i}} \sum_{i}^{N_{i}}\left(\frac{B(i)-B_{0}(i)}{B(i)}\right)^{2}
$$

using GA, where, $B_{0}$ and $B$ denotes the magnetic flux density shown in Fig. 6 and that computed by the Preisach model. The resultant parameters are summarized in the left column of Table III. Based on this result, $L_{d c}-I_{\text {bias }}$ characteristic is computed using the method mentioned in II.B.

\section{B. Identified Results}

The $L_{a c}-I_{\text {peak }}$ and $L_{d c}-I_{\text {bias }}$ characteristics computed from the measured data shown in Fig. 6 and fitted by the proposed method mentioned in III are plotted in Fig. 7 and Fig. 8. The parameters identified from the inductance characteristics are summarized in the right column of Table III. Although there are small discrepancies for small input currents in $L_{a c}-I_{\text {peak }}$ characteristic, satisfactory coincidence is obtained. The convergence history of GA for solving problem (9) is shown in Fig. 9. We can find in Fig. 9 that the cost function $F$ becomes sufficiently small after 1000 generations. The initial magnetization curve and the $\mathrm{BH}$ loops identified from the inductance characteristics shown in Figs. 7 and 8 are plotted with the original curves obtained from the measured data in Fig. 6 and the Preisach model in Fig. 10 and 11. It can be seen that both are in good agreement. Figure 12 shows the hysteresis loss with respect to the amplitude of magnetic flux density. The hysteresis loss is overestimated by the proposed method in comparison with the measured value. This discrepancy comes from the errors in the $\mathrm{BH}$ loops shown in Fig. 11; the ratio $S_{1} / S_{2}$, where $S_{1}$ and $S_{2}$ denote the area of the measured and identified minor loops, respectively, is almost the same as the ratio of the measured and computed hysteresis losses shown in Fig. 12.

\section{Discussions}

The above-mentioned method is based on the assumption that the $\mathrm{BH}$ characteristics can be described by the Preisach model with the distribution function (7). If the core material does not obey this model, the present identification would be unsuccessful. If the distribution function of the material can be described by an explicit function of parameters, the proposed method can be extended to identify the parameters. The extension of the proposed method to identification of implicit distribution functions is remained as an open question.

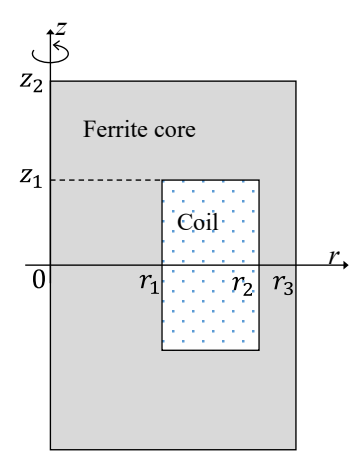

TABLE I

\begin{tabular}{lr}
\multicolumn{2}{c}{ TNDUCTOR PARAMETERS } \\
\hline \hline$r_{1}[\mathrm{~mm}]$ & 0.45 \\
$r_{2}[\mathrm{~mm}]$ & 0.65 \\
$r_{3}[\mathrm{~mm}]$ & 1.00 \\
$z_{1}[\mathrm{~mm}]$ & 0.35 \\
$z_{2}[\mathrm{~mm}]$ & 0.75 \\
Number of coil turns & 8 \\
\hline \hline
\end{tabular}

Fig. 5. Analysis model

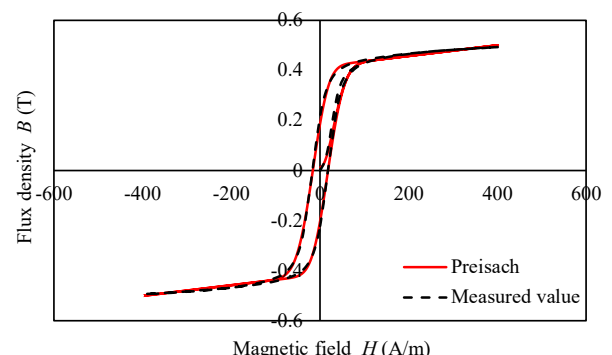

Fig. 6. Magnetic property of the ferrite core

TABLE II

\begin{tabular}{lr}
\multicolumn{2}{c}{ ANALYSIS CONDITIONS } \\
\hline \hline$H_{s}[\mathrm{~A} / \mathrm{m}]$ & 400 \\
$\Delta H[\mathrm{~A} / \mathrm{m}]$ & 4 \\
$N_{p}$ & 200 \\
Population of GA & 400 \\
Generation of GA & 1000 \\
\hline \hline
\end{tabular}

TABLE III

PREISACH PARAMETERS

\begin{tabular}{lrr}
\hline \hline & identified from Fig.6 & $\begin{array}{r}\text { identified from } L \\
\text { characteristics }\end{array}$ \\
\hline$A$ & 0.81 & 0.82 \\
$\sigma_{1}$ & 23.44 & 29.52 \\
$\sigma_{2}$ & 24.50 & 21.60 \\
$\mu_{r}^{\text {out }}$ & 170.21 & 173.06 \\
\hline \hline
\end{tabular}

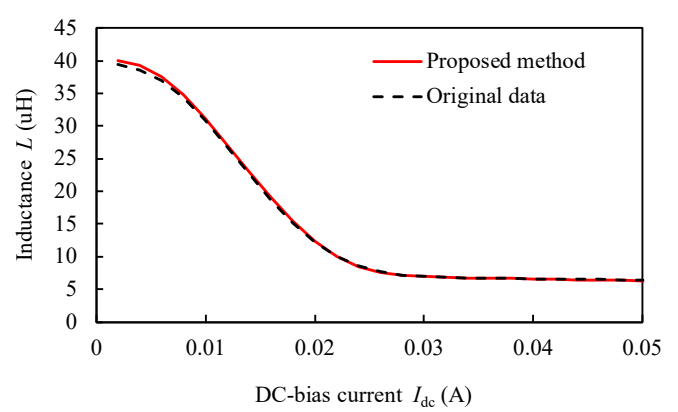

Fig. 7. $L_{d c}-I_{\text {bias }}$ characteristic

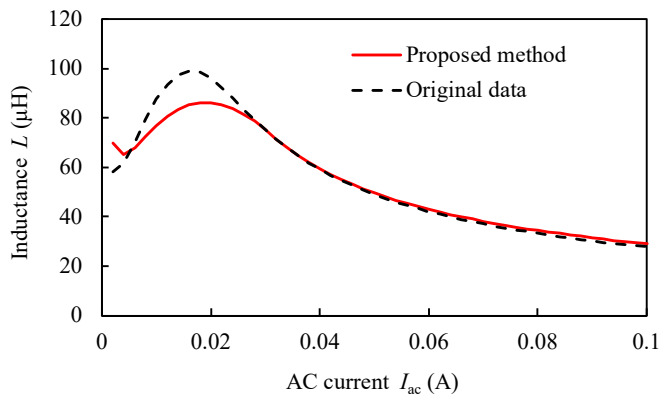

Fig. 8. $L_{a c}-I_{\text {peak }}$ characteristic 


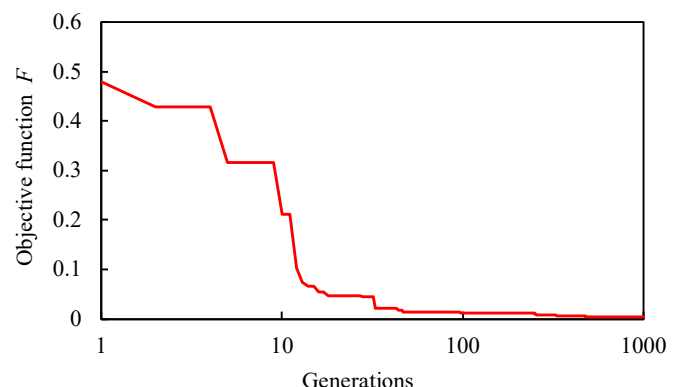

Fig. 9. Convergence history of GA

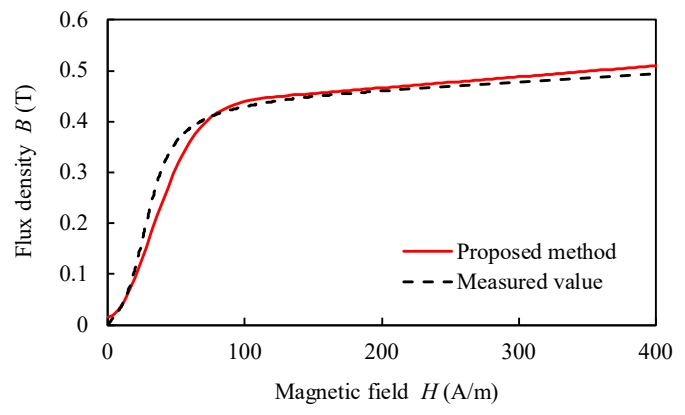

Fig. 10. Identified initial magnetization curve

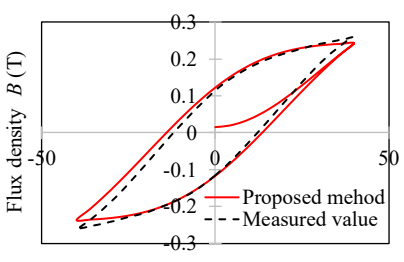

Magnetic field $H(\mathrm{~A} / \mathrm{m})$

(a) $H_{m}=40$

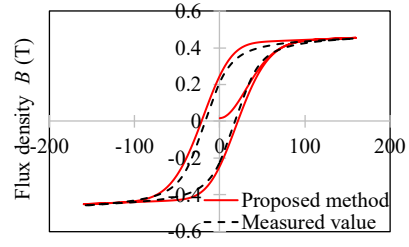

Magnetic field $H(\mathrm{~A} / \mathrm{m})$

(c) $H_{m}=160$

Fig. 11. Identified BH loops, where $H_{m}$ denotes the amplitude of $H$

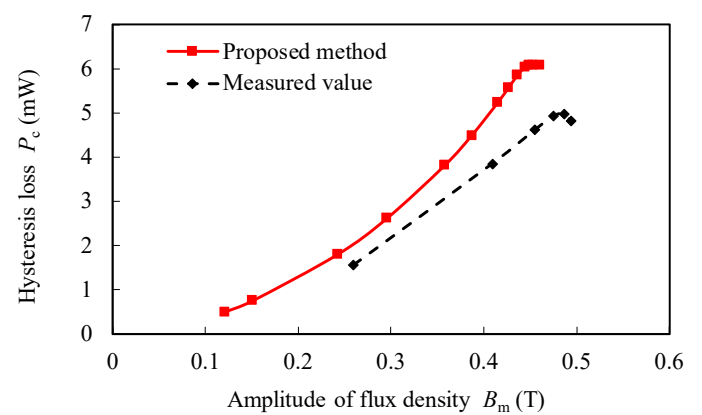

\section{Uniqueness of Solution}

We consider here if the $\mathrm{BH}$ characteristics can be uniquely determined from the inductance characteristics. When assuming the distribution function of (7), the $\mathrm{BH}$ characteristics are described by the parameters $\left(A, \sigma_{1}, \sigma_{2}, \mu_{r}^{\text {out }}\right)$. To verify if these parameters are uniquely determined from the inductance characteristics, we performed additional five identifications starting from different initial populations generated randomly. The identified parameters and the final value of $F$ are summarized in Table IV. It can be concluded from these results that there exists uniqueness to the solution at least for the data considered here. The theoretical proof of the uniqueness is remained for a future work.

\section{CONCLUSION}

We have proposed a new method to identify the magnetization characteristics of ferrite used for the magnetic core of an inductor from the inductance characteristics. This approach is useful because special measurement instruments for $\mathrm{BH}$ loops are not needed. The $\mathrm{BH}$ characteristics are computed from a measured major loop. The inductance characteristics are obtained from those $\mathrm{BH}$ data. Then, the $\mathrm{BH}$ characteristics have been identified from the inductance characteristics. The identification result is satisfactory. The uniqueness for the identification result is numerically verified. The extension of the proposed method to implicit distribution functions and theoretical proof of the uniqueness have been remained as open questions.

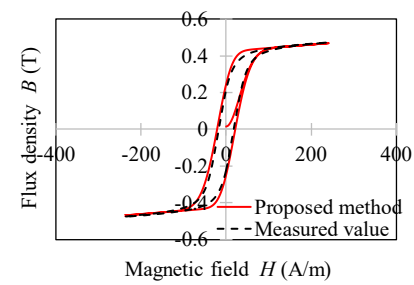

(d) $H_{m}=240$

\section{REFERENCES}

[1] K. Watanabe, et al., "Optimization of Inductors Using Evolutionary Algorithms and Its Experimental Validation," IEEE Transactions on Magnetics, vol. 46 no. 8, pp. 3393-3396, 2010.

[2] T. Matsuo and M. Shimasaki, "An identification method of play model with input-dependent shape function," IEEE Transactions on Magnetics, vol. 41, no. 10, pp. 3112-3114, 2005.

[3] Y. Takeda, et al., "Iron Loss Estimation Method for Rotating Machines Taking Account of Hysteretic Property," IEEE Transactions on Magnetics, vol. 51, no. 3, pp. 1-4, 2015.

[4] IWATSU, "B-H Analyzer SY-8218 / SY-8219", https://www.iti.iwatsu.co.jp/en/products/sy/sy8218_top_e.html.

[5] F. Preisach, "Über die Magnetische Nachwirkung," Zeitschrift für Physik, vol. 94, pp. 277-302, 1935.

[6] G. Bertotti, "Hysteresis in Magnetism," Academic Press, 1998

[7] E. Della Torre., "Magnetic Hysteresis," IEEE Press, 1999.

Fig. 12. Hysteresis loss with respect to amplitude of magnetic flux density

TABLE IV

IDENTIFIED PARAMETERS AND RESIDUAL

\begin{tabular}{lrrrrrr}
\hline \hline & $\# 1$ & $\# 2$ & $\# 3$ & $\# 4$ & $\# 5$ & $\# 6$ \\
\hline$A$ & 0.82 & 0.82 & 0.81 & 0.83 & 0.82 & 0.81 \\
$\sigma_{1}$ & 29.52 & 29.66 & 28.98 & 30.06 & 29.43 & 29.42 \\
$\sigma_{2}$ & 21.60 & 21.61 & 21.56 & 21.65 & 21.45 & 21.53 \\
$\mu_{r}^{\text {out }}$ & 173.06 & 173.94 & 173.27 & 173.83 & 173.95 & 174.30 \\
Final $F$ & $3.99 \times 10^{-3}$ & $3.99 \times 10^{-3}$ & $3.72 \times 10^{-3}$ & $4.39 \times 10^{-3}$ & $3.89 \times 10^{-3}$ & $3.86 \times 10^{-3}$ \\
\hline \hline
\end{tabular}

